\title{
Interceptação em plantio de Araucaria angustifolia (Bertol.) Kuntze (Araucariaceae) no Parque Estadual da Serra do Mar, núcleo Cunha, SP.
}

\author{
Rita de Cássia Sousa ${ }^{1}$ \\ Maurício Ranzini ${ }^{2}$ \\ Francisco Carlos Soriano Arcova ${ }^{3}$ \\ Valdir de $\mathrm{Cicco}^{4}$ \\ Carla Daniela Câmara ${ }^{5}$ \\ ${ }^{1}$ Instituto Florestal do Estado de São Paulo - Divisão de Dasonomia \\ Rua do Horto, 931 - São Paulo, SP - 02377-000 \\ rita-sousa@uol.com.br \\ ${ }^{2}$ Instituto Florestal do Estado de São Paulo - Divisão de Dasonomia \\ Rua do Horto, 931 - São Paulo, SP - 02377-000 \\ ranzini@if.sp.gov.br \\ ${ }^{3}$ Instituto Florestal do Estado de São Paulo - Divisão de Dasonomia \\ Rua do Horto, 931 - São Paulo, SP - 02377-000. \\ arcova@if.sp.gov.br \\ ${ }^{4}$ Instituto Florestal do Estado de São Paulo. Divisão de Dasonomia \\ Rua do Horto, 931 - São Paulo, SP - 02377-000. \\ valdircicco@if.sp.gov.br \\ ${ }^{5}$ Universidade Tecnológica Federal do Paraná \\ Avenida Brasil 4232 - Medianeira, PR - 85884-000. \\ carladanielacamara@ig.com.br
}

\begin{abstract}
Due to the importance of the species Araucaria angustifolia (Bertol.) Kuntze and on account of its presence in the "Núcleo Cunha", it was decided to study its influence on the process of rainfall redistribution. Daily throughfall (Tr) and stemflow of trees (Et) in a plot of $600 \mathrm{~m}^{2}$, equipped with 15 rain gauges and devices for collecting stemflow water on 19 trees were measured. A rain gauge installed in the open area located near the plot provided the rainfall data in the open $(\mathrm{Pa})$. The interception $(\mathrm{I})$ was estimated by the equation: $\mathrm{I}=\mathrm{Pa}-(\mathrm{Et}+$ Tr). The experimental period covered from October 2008 to March 2009. Sixty-eight rain events were recorded, resulting in $1,565.9 \mathrm{~mm}$ of precipitation in the open, and $163.4 \mathrm{~mm}$ were intercepted by the canopy. The gross precipitation is directly proportional to the rainfall in the open, since the intercept tends to decrease with the increase of rainfall classes. Negative interception events were recorded, showing another water entrance in the system.
\end{abstract}

Palavras-chave: Araucaria angustifolia (Bertol.) Kuntze, gross precipitation, interception, throughfall, stemflow, precipitação efetiva, interceptação, transprecipitação, escoamento pelo tronco. 


\section{INTRODUÇÃO}

A influência da vegetação no recebimento e redistribuição das chuvas é significativa, dentro do contexto do balanço hídrico de uma determinada área, isto é, diferentes coberturas florestais influenciam na recarga hídrica do solo.

Os processos envolvidos na redistribuição das chuvas são: a interceptação, que consiste na fração da precipitação retida nas folhas e ramos das árvores, retornando à atmosfera como vapor; a transprecipitação (ou precipitação interna) que compreende a porção da chuva que atravessa o dossel, chegando ao solo na forma de gotas; e o escoamento pelo tronco; a precipitação efetiva é a soma desses dois últimos fluxos hídricos (Arcova et al., 2003).

O Instituto Florestal desenvolve pesquisas sobre diferentes aspectos do ciclo hidrológico em microbacias experimentais no Laboratório de Hidrologia Florestal Engenheiro Agrônomo Walter Emmerich, localizado no núcleo Cunha do Parque Estadual da Serra do Mar. Os estudos concentram-se basicamente em microbacias recobertas por Mata Atlântica, a fitofisionomia predominante da região. Porém há nas proximidades considerável ocorrência da espécie Araucaria angustifolia (Bertol.) Kuntze, o "pinheiro do Paraná", sendo a localidade uma grande produtora de sua semente, o pinhão.

O pinheiro é nativo do Brasil, com ocorrência natural em Floresta Ombrófila Mista, em áreas contínuas no sul do país, e na região sudeste são encontrados fragmentos deste bioma em locais com altitudes elevadas (Souza, 2008). Apesar de ocupar extensas áreas, a sua exploração indiscriminada colocou-a nas listas oficiais das espécies ameaçadas de extinção no território paulista (Secretaria do Meio Ambiente - SMA, 2004), no Brasil (Biodiversitas, 2006) e no mundo (International Union for Conservation of Nature - IUCN, 2006).

Em função da importância econômica desta espécie para a comunidade local e, também, pelo fato de sua expressiva presença no núcleo Cunha, decidiu-se por estudar a influência dessa cobertura florestal sobre a redistribuição da água das chuvas pelo dossel.

Informações acerca dos processos hidrológicos em vegetação de araucárias podem gerar conhecimentos que subsidiem projetos de conservação da espécie. Neste sentido, o presente estudo tem como objetivo estimar a interceptação em um plantio de A. angustifolia, e determinar a participação da transprecipitação e do escoamento pelo tronco na redistribuição das chuvas pelas copas das árvores.

\section{MATERIAL E MÉTODOS}

A área experimental está inserida no Laboratório de Hidrologia Florestal Eng. Agr. Walter Emmerich, pertencente ao núcleo Cunha do Parque Estadual da Serra do Mar, com uma extensão de 2.854 ha, localiza-se nas cabeceiras do rio Paraibuna e na margem direita do rio Paraíba do Sul, entre os paralelos $23^{\circ} 13^{\prime} 28^{\prime \prime}$ e $23^{\circ} 16^{\prime} 10^{\prime \prime}$ de latitude sul e os meridianos $45^{\circ} 02^{\prime} 53^{\prime}$ ' e $45^{\circ} 05^{\prime} 15^{\prime \prime}$ de longitude oeste de Greenwich, com altitudes entre $1.040 \mathrm{~m}$ a $1.160 \mathrm{~m}$ (Luiz, 2008).

O sistema fisionômico-ecológico classifica a área como Floresta Ombrófila Densa Montana, isto é, situada na serra com latitude entre $16^{\circ}$ a $24^{\circ} \mathrm{S}$ e altitude entre $500 \mathrm{~m}$ a $1.500 \mathrm{~m}$ (Veloso, 1991).

Conforme Luiz (2008), em um período de 25 anos de observações no núcleo Cunha, o tipo climático predominante foi o $\mathrm{Cwb}$, segundo a classificação de Köppen, ou seja, clima temperado chuvoso e moderadamente quente, com preponderância de chuvas em verões brandamente quentes.

As precipitações anuais são elevadas, resultantes dos efeitos orográficos da Serra do Mar. A precipitação média anual de 16 anos de observação é de $2.205 \mathrm{~mm}$, com valores extremos de $1.769 \mathrm{~mm}$ e $3.040 \mathrm{~mm}$ (Cicco, 2004). O período chuvoso estende-se de outubro a março e 
o período menos chuvoso, compreende os meses de abril a setembro. A temperatura média anual do ar é de $16,8^{\circ} \mathrm{C}$ raramente ultrapassando os $20^{\circ} \mathrm{C}$ (Luiz, 2008). A umidade relativa média mensal do ar está entre $80 \%$ e $85 \%$ para o período chuvoso, e inferior a $80 \%$ para o restante do ano, com ocorrência frequente de nevoeiros densos nos períodos crepuscular e noturno (Arcova, 1996).

A área experimental abrange $2.000 \mathrm{~m}^{2}$, mas o local efetivo de medição é de $600 \mathrm{~m}^{2}$ $(20 \times 30 \mathrm{~m}) \mathrm{com}$ uma faixa de $10 \mathrm{~m}$ mantida ao redor da parcela, com o objetivo de atenuar o efeito de borda sobre a mesma. O principal componente do dossel são espécimes de A. angustifolia, um plantio com 29 anos, contendo 19 árvores, com diâmetros de $9 \mathrm{~cm}$ a $47 \mathrm{~cm}$.

O período experimental abrange os meses de outubro de 2008 a março de 2009 (período chuvoso). A precipitação no aberto, isto é, a precipitação que ocorre fora do povoamento de araucária, foi medida por um pluviômetro instalado a $100 \mathrm{~m}$ da parcela.

A transprecipitação foi amostrada em uma matriz de 31 pontos, constituída de seis linhas espaçadas por cinco metros, com os pontos distantes $3,3 \mathrm{~m}$ entre si. Em cada ponto foi instalado um dispositivo feito de estaca de madeira acoplada a uma base de cano de PVC de 15 polegadas. Em número de quinze, os pluviômetros foram remanejados semanalmente, a partir de sorteio dos 31 pontos preestabelecidos.

Para a quantificação do escoamento pelo tronco foram confeccionados coletores de espuma de poliuretano, conforme metodologia proposta por Likens e Eaton (1970), os quais foram instalados nas 19 árvores da parcela.

As quantificações de precipitação no aberto, de transprecipitação e do escoamento pelo tronco foram realizadas diariamente, quando do acontecimento de evento chuvoso.

A transprecipitação correspondeu à média das quantidades de chuva nos 15 pluviômetros. A quantidade de água escoada pelo tronco foi determinada a partir do somatório das coletas de escoamento pelo tronco das 19 árvores. Utilizou-se a superfície total da parcela para a conversão em unidade de milímetros, conforme a equação 1:

$$
\mathrm{Et}(\mathrm{mm})=\left[\frac{(\mathrm{Et}(\mathrm{mL}))}{1000}\right] \div \mathrm{A}
$$

Et $(\mathrm{mm})=$ escoamento pelo tronco;

$\mathrm{Et}(\mathrm{mL})=$ volume total de água dos coletores de escoamento pelo tronco; e

$\mathrm{A}=$ área da parcela em metros quadrados.

Para estimativa da interceptação foi empregada a equação 2 proposta por Helvey e Patric (1965):

$$
\mathrm{I}=\mathrm{Pa}-(\mathrm{Tr}+\mathrm{Et})
$$

$\mathrm{I}=$ interceptação $(\mathrm{mm})$;

$\mathrm{Pa}=$ precipitação no aberto $(\mathrm{mm})$;

$\operatorname{Tr}=$ transprecipitação $(\mathrm{mm})$; e

$\mathrm{Et}=$ escoamento pelo tronco $(\mathrm{mm})$.

\section{RESULTADOS E DISCUSSÃO}

Durante experimento foram registrados 68 eventos de chuvas, resultando em 1.565,9 mm de precipitação no aberto, equivalente a $71 \%$ da precipitação média anual no laboratório. Esse valor parece ser elevado, porém tais dados são relativos a seis meses do período chuvoso, o 
que ajuda a explicar o resultado apresentado. Os valores mensais dos componentes analisados podem ser visualizados na Tabela 1.

TABELA 1. Valores correspondentes à precipitação no aberto $(\mathrm{Pa})$, transprecipitação (Tr), escoamento pelo tronco (Et), precipitação efetiva (Pe) e interceptação (I) em (mm) e (\%) em relação à precipitação no aberto.

\begin{tabular}{cccccccccc}
\hline \multirow{2}{*}{ Mês/ Ano } & Pa & \multicolumn{2}{c}{ Tr } & \multicolumn{2}{c}{ Et } & \multicolumn{2}{c}{ Pe } & \multicolumn{2}{c}{ I } \\
& $\mathbf{( m m )}$ & $(\mathbf{m m})$ & $\mathbf{\%}$ & $\mathbf{( m m )}$ & $\mathbf{\%}$ & $\mathbf{( m m )}$ & $\mathbf{\%}$ & $\mathbf{( m m )}$ & $\boldsymbol{\%}$ \\
\hline out./ 2008 & 166,0 & 143,0 & 86,1 & 0,3 & 0,2 & 143,3 & 86,3 & 22,7 & 13,7 \\
nov./ 2008 & 273,4 & 247,8 & 90,6 & 0,1 & 0,1 & 247,9 & 90,7 & 25,5 & 9,3 \\
dez./ 2008 & 217,3 & 194,3 & 89,4 & 0,2 & 0,1 & 194,5 & 89,5 & 22,8 & 10,5 \\
jan./ 2009 & 333,3 & 289,6 & 86,9 & 0,7 & 0,2 & 290,3 & 87,1 & 43,0 & 12,9 \\
fev./ 2009 & 297,0 & 271,5 & 91,4 & 0,6 & 0,2 & 272,1 & 91,6 & 24,9 & 8,4 \\
mar./ 2009 & 278,9 & 254,1 & 91,1 & 0,3 & 0,1 & 254,4 & 91,2 & 24,5 & 8,8 \\
\hline TOTAL & $\mathbf{1 . 5 6 5 , 9}$ & $\mathbf{1 . 4 0 0 , 3}$ & $\mathbf{8 9 , 4}$ & $\mathbf{2 , 2}$ & $\mathbf{0 , 2}$ & $\mathbf{1 . 4 0 2 , 5}$ & $\mathbf{8 9 , 6}$ & $\mathbf{1 6 3 , 4}$ & $\mathbf{1 0 , 4}$ \\
\hline
\end{tabular}

Da precipitação no aberto, $1.400,3 \mathrm{~mm}$ de água que chega efetivamente ao solo, foi pelo processo de transprecipitação, sendo essa a principal via de entrada de água no sistema. Já o escoamento pelo tronco contribui com apenas $2,2 \mathrm{~mm}$, isto é, $0,2 \%$ da precipitação no aberto.

Arcova et al. (2003), no mesmo local de estudo, porém em Mata Atlântica, verificaram que a transprecipitação, o escoamento pelo tronco e a interceptação corresponderam a 81,2\%, 0,2\% e 18,6\% da precipitação no aberto; isto é, a Floresta Ombrófila Densa interceptou 8\% a mais que o plantio de araucária.

Lima e Nicolielo (1983) estimaram a interceptação em duas espécies de Pinus, e chegaram a valores similares ao encontrado nesse trabalho, aproximadamente $12 \%$ das precipitações no aberto são interceptados pelas copas dos pinheiros.

O maior percentual mensal da interceptação foi verificado em outubro, correspondendo a $13,7 \%$ da precipitação no aberto. Quando a precipitação é pequena observa-se maior percentual de chuva interceptada pelas copas das araucárias, conforme a Tabela 2, onde as precipitações no aberto foram divididas por classes.

TABELA 2. Classe de chuva, frequência e valores médios por classe de chuva, da precipitação no aberto $(\mathrm{Pa})$ em $(\mathrm{mm})$, precipitação efetiva (Pe) e interceptação (I) em (mm) e (\%) em relação à precipitação no aberto.

\begin{tabular}{ccccccc}
\hline $\begin{array}{c}\text { Classes } \\
(\mathbf{m m})\end{array}$ & \multirow{2}{*}{ Frequência } & $\mathbf{P a}$ & \multicolumn{2}{c}{ Pe } & \multicolumn{2}{c}{ I } \\
\cline { 3 - 7 } & & $\mathbf{( m m})$ & $(\mathbf{m m})$ & $\mathbf{\%}$ & $\mathbf{( m m})$ & \% \\
\hline$<5$ & 16 & 2,6 & 1,8 & 69,2 & 0,8 & 30,8 \\
$5-10$ & 9 & 8,0 & 5,9 & 73,8 & 2,1 & 26,2 \\
$10-20$ & 18 & 15,2 & 13,4 & 88,2 & 1,8 & 11,8 \\
$20-30$ & 6 & 25,2 & 22,4 & 88,9 & 2,8 & 11,1 \\
$30-40$ & 3 & 32,5 & 30,4 & 93,5 & 2,1 & 6,5 \\
$40-50$ & 4 & 44,5 & 40,8 & 91,7 & 3,7 & 8,3 \\
$>50$ & 12 & 62,7 & 57,5 & 91,7 & 5,2 & 8,3 \\
\hline
\end{tabular}

Os valores de precipitação efetiva são diretamente proporcionais aos da precipitação no aberto. Já a interceptação tende a decrescer com o aumento das classes de chuva, como também verificado por Castro et al. (1983), Coelho Neto et al. (1986) e Thomaz (2005). 
O valor máximo de interceptação é de $30,8 \%$ da precipitação no aberto, inferior ao encontrado por Thomaz (2005) em estudo com A. angustifolia no município de Guarapuava (PR), que estimou até 50\% de interceptação, porém para um ano hídrico completo.

As relações entre a transprecipitação, o escoamento pelo tronco e a interceptação em função da precipitação no aberto, podem ser visualizadas na Figura 1. A transprecipitação pode ser explicada pela precipitação no aberto em função do alto coeficiente de determinação $\left(\mathrm{R}^{2}=99,4 \%\right)$, Figura 1A. O mesmo foi observado em outras coberturas vegetais, como em cerrado (Lima e Nicolielo, 1983), em mata ciliar (Lima e Leopoldo, 2000) e na Mata Atlântica (Arcova et al. 2003).

$\mathrm{O}$ escoamento pelo tronco apresentou $\mathrm{R}^{2}$ de $82,5 \%$, o que representa uma elevada relação com a precipitação no aberto (Figura 1B). Arcova et al. (2003) no mesmo local de estudo, porém em Mata Atlântica, chegaram a valores próximos ao apresentado neste trabalho.
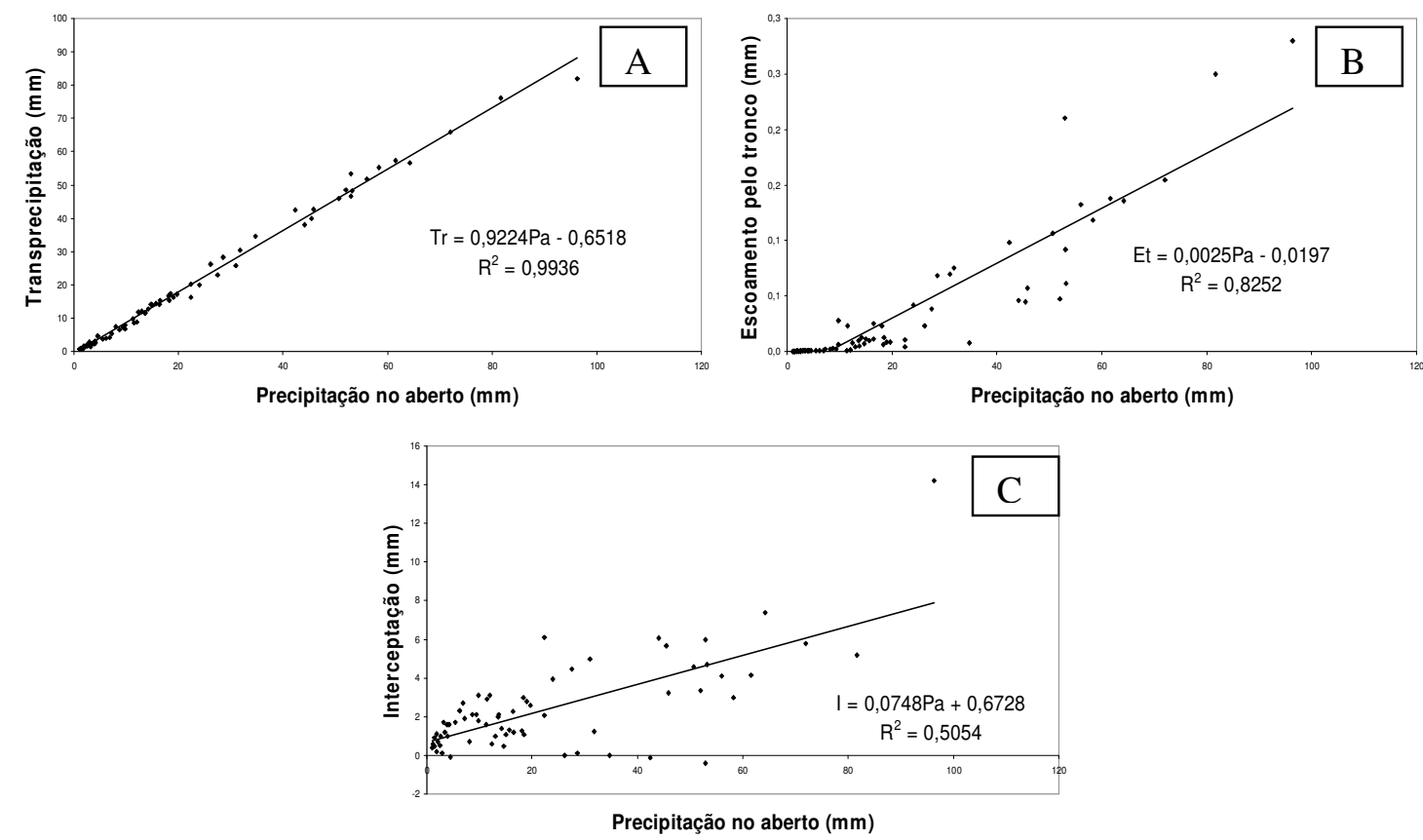

FIGURA 1. Relação entre a transprecipitação (A), escoamento pelo tronco (B) e interceptação (C) e a precipitação no aberto.

A relação entre a interceptação e a precipitação no aberto mostrou-se fraca, evidenciada pelo baixo coeficiente de determinação $\left(\mathrm{R}^{2}=50,5 \%\right)$, Figura 1C. Arcova et al. (2003) verificaram resultados similares para a mesma estação do ano.

Pelo fato da transprecipitação e do escoamento pelo tronco apresentarem fortes relações com a precipitação no aberto, é possível estimar seus valores a partir das equações estabelecidas.

Na Figura 1C, cinco valores de interceptação são iguais ou menores que zero. Esses eventos podem indicar outra entrada de água no sistema, sendo uma das hipóteses a ocorrência de neblina no local, como relatado por Anido (2002).

\section{CONCLUSÕES}

As maiores interceptações pelas copas das araucárias ocorreram quando das precipitações de menores magnitudes. Já a precipitação efetiva é diretamente proporcional à precipitação no aberto. 
Os percentuais de transprecipitação, escoamento pelo tronco e interceptação em relação à precipitação no aberto foram: $89,4 \%, 0,2 \%, 10,4 \%$, respectivamente.

A interceptação em função da precipitação no aberto apresentou um baixo coeficiente de determinação. As coletas continuam a ser realizadas para que se possam avaliar os processos de um ano hídrico completo.

\section{REFERÊNCIAS BIBLIOGRÁFICAS}

ANIDO, N. M. R. Caracterização hidrológica de uma microbacia experimental visando identificar indicadores de monitoramento ambiental. 2002. Piracicaba. 69 p. Dissertação (Mestrado em Recursos Florestais) - Escola Superior de Agricultura “Luiz de Queiroz”, Universidade de São Paulo. 2002.

ARCOVA, F. C. S. Balanço hídrico, características do deflúvio e calibragem de duas microbacias hidrográficas na Serra do Mar, SP. Piracicaba. 1996. 155 p. Dissertação (Mestrado em Ciências Florestais) - Escola Superior de Agricultura “Luiz de Queiroz”, Universidade de São Paulo. 1996.

ARCOVA, F. C. S.; CICCO, V.; ROCHA, P. A. B. Precipitação efetiva e interceptação das chuvas por floresta de mata atlântica em uma microbacia experimental em Cunha - São Paulo. Revista Árvore, Viçosa, v. 27, n. 2, p. 257-262, 2003.

BIODIVERSITAS. Lista oficial de espécies ameaçadas de extinção no Brasil. 2006. Disponível em: <http://www.biodiversitas.org.br/florabr/grupo3fim.asp>. Acesso em: 23 jun. 2009.

CASTRO, P. S.; VALENTE, O. F.; COELHO, D. T.; RAMALHO, R. S.Interceptação da chuva por mata natural secundária na região de Viçosa, MG. Revista Árvore, Viçosa, MG, v. 7, n. 1, p. 76 - 89, 1983.

COELHO NETTO, A. L.; SANCHE, M.; PEIXOTO, M. N. O. Precipitação e interceptação florestal em ambiente tropical montanhoso, Rio de Janeiro. Revista Brasileira de Engenharia, Rio de Janeiro, v. 4, n. 2, p. 55- 71, 1986.

CICCO, V. Análise de séries temporais hidrológicas em microbacia com cobertura vegetal natural de Mata Atlântica, Cunha-SP. 2004. 149 p. Dissertação (Mestrado em Geografia) - Faculdade de Filosofia, Letras e Ciências Humanas, Universidade de São Paulo. 2004.

HELVEY, J. D.;PATRIC, J. H. Canopy and litter interception of rainfall by hardwoods of eastern United States. Water resources research. v. 1, p. $193-206,1965$.

INTERNATIONAL UNION FOR CONSERVATION OF NATURE - IUCN. Lista vermelha de espécies ameaçadas de extinção da união internacional para a conservação da natureza. 2006. Disponível em: <http://www.iucnredlist.org/> Acesso em: 23 de jun. de 2009.

LIKENS, G. E.; EATON, J. S. A polyurethane stemflow collector for trees and shrubs. Ecology, v. 51, n. 5, p. $938-939,1970$.

LIMA, P. R.; LEOPOLDO, P. R. Quantificação de componentes hidrológicos de uma mata ciliar, através do modelo de balanço de massas. Revista Árvore, v. 24, n. 3, p. 241-252, 2000.

LIMA, W. P.; NICOLIELO, N. Precipitação efetiva e interceptação em florestas de pinheiros tropicais e em reserva de cerradão. Revista IPEF, Piracicaba, n. 24, p. 43 - 46, 1983.

LUIZ, R. A. F. Classificação climática do Núcleo Cunha do Parque Estadual da Serra do Mar, Cunha SP. 2008. 103 p. Trabalho de Graduação Individual - Faculdade de Filosofia, Letras e Ciências Humanas, Universidade de São Paulo. 2008.

SECRETARIA DO MEIO AMBIENTE - SMA. Resolução SMA 48, de 21 de Setembro. Lista oficial de espécies ameaçadas de extinção no Estado de São Paulo. 2004. Disponível em: <http://www.ibot.sp.gov.br/legislacao/legislacao.htm.> Acesso em: 23 de jun. de 2009.

SOUZA, R. P. M. Estrutura da comunidade arbórea de trechos de florestas de Araucaria no estado de São Paulo, Brasil. 2008. 102 p. Dissertação (Mestrado em Recursos Florestais) - Escola Superior de Agricultura "Luiz de Queiroz", Universidade de São Paulo. 2008.

THOMAZ, E. L. Avaliação de interceptação e precipitação interna em capoeira e floresta secundária em Guarapuava - PR. Geografia, v. 14, n. 1, p. 47 - 60, 2005. 
Anais II Seminário de Recursos Hídricos da Bacia Hidrográfica do Paraíba do Sul: Recuperação de Áreas Degradadas Serviços Ambientais e Sustentabilidade, Taubaté, Brasil, 09-11 dezembro 2009, IPABHi, p. 117-124.

(doi:10.4136/serhidro.16)

VELOSO, H. P.; FILHO, A. L. R. R.; LIMA, J. C. A. Classificação da vegetação brasileira, adaptada a um sistema universal. Rio de Janeiro: Departamento de Recursos Naturais e Estudos Ambientais. 1991. 124 p. 